\title{
A comparison of the magnetic properties of polysaccharide iron complex (PIC) and ferritin
}

\author{
M-E.Y. Mohie-Eldin ${ }^{\text {a }}$, R.B. Frankel ${ }^{\text {b }}$, L. Gunther ${ }^{\text {a }}$ \\ a Department of Physics and Astronomy, Tufts University, Medford, MA 02155, USA \\ ${ }^{b}$ Physics Department, California Polytechnic State University, San Luis Obispo, CA 93407, USA
}

\begin{abstract}
The synthetic polysaccharide iron complex (PIC) molecule has been suggested as a 'biomimic', i.e. a counterpart, to the naturally occurring biological molecule ferritin with respect to its magnetic properties based on the identification of ferrihydrite as the major mineral in both. Magnetization measurements were used to investigate the magnetic properties of PIC in relation to those of ferritin, as well as to identify differences in such properties between naturally occurring ferritin, which we designate here as Ferritin I, and ferritin with an artificially high content of $\mathrm{Fe}^{2+}$ ions bound to its core, which is designated here as Ferritin II. The anisotropy constants $K$, blocking temperatures $T_{\mathrm{B}}$, magnetic moments $m$ per particle, and number of magnetic moments 'spins' per particle $N_{\mathrm{sp}}$ were found to fit the following relations for PIC and ferritin: $K$ (Ferritin I) $<K(\mathrm{PIC}) \leq K$ (Ferritin II), $T_{\mathrm{B}}$ (Ferritin I) $<T_{\mathrm{B}}\left(\right.$ Ferritin II) $<T_{\mathrm{B}}(\mathrm{PIC}), m($ Ferritin II $) \sim m\left(\right.$ Ferritin I) $<m(\mathrm{PIC}), N_{\mathrm{sp}}($ Ferritin II $)=N_{\mathrm{sp}}\left(\right.$ Ferritin I) $<N_{\mathrm{sp}}(\mathrm{PIC})$. The magnetic moment per Fe ion was found to be smaller in PIC than Ferritin II due to a stronger antiferromagnetic interaction between the Fe ions of PIC. Susceptibility measurements indicated the existence of superantiferromagnetism in PIC and Ferritin I and also showed that most $\mathrm{Fe}^{2+}$ ions in Ferritin II are bound to its core surface. The enhanced values of $K$ and $T_{\mathrm{B}}$ as well as the reduced effect of superantiferromagnetism and the antiferromagnetic interaction between the molecules at low temperatures for the Ferritin II indicates the importance of the surface magnetic moments in dominating the magnetic behavior of both PIC and ferritin (Ferritin I).
\end{abstract}

\section{Introduction}

Naturally occurring biological materials provide well-defined model systems which can be used to test theoretical ideas concerning fine particle magnetism. The magnetic properties of these materials provide a convenient and important diagnostic for understanding their structure and properties, particularly when there exist anomalies pertaining to one or both as well as identifying important differences among related materials. Occasionally, novel synthetic materials that can be easily modified are produced, which 'biomimic' the biological material in some or all of its properties. These synthetic materials represent a great aid in understanding such anomalies by serving as useful models of the naturally occurring biological material. Fast (response time 
$\leq 10^{-7}$ s) short-range probing techniques such as Mössbauer spectroscopy and X-ray diffraction have suggested [1] that the polysaccharide iron complex (PIC) molecule is such a 'biomimic' of the naturally occurring biological molecule 'ferritin', based on the identification of ferrihydrite as the major mineral in both. The iron present in both was determined to be the high-spin $\mathrm{Fe}^{3+}$ only. However, no study of PIC's magnetic properties has been carried out for comparison with those of ferritin, although several studies have been reported on the naturally occurring ferritin's magnetic properties [2-9].

In this paper we present results of an investigation of the magnetic behavior of PIC and ferritin using different methods to determine their regions of superparamagnetism, maximum blocking temperatures, magnetic moments as well as number of uncompensated spins per core (particle), anisotropy constants given the particles' volumes, and their Néel temperatures. Basically, we want to know if PIC and ferritin give a consistent picture of small antiferromagnetic particles, because our primary goal is to find whether the synthetic PIC molecule is a useful 'biomimic' model in investigating and comparing the recently observed [10] Lamb-Mössbauer $f$-factor anomaly in ferritin. This anomaly reveals itself in the sudden drop in the $f$-factor with increasing temperature around (mammalian) ferritin's blocking temperature $T_{\mathrm{B}}=37 \mathrm{~K}$, which is also the temperature above which the hyperfine structure, in ferritin's Mössbauer spectrum, disappears and is replaced by a quadrupole doublet. Therefore a synthetic molecule which is easily handled and which 'biomimics' ferritin's properties and behavior would be quite useful in studying this anomaly. For example, one of the initial questions such a 'biomimic' can help us answer is whether the simultaneous disappearance (appearance) of the hyperfine structure near the blocking temperature with the anomalous decrease (increase) of the $f$-factor is coincidental or related. However, such a 'biomimic' should, as a first requirement, possess a different blocking temperature than ferritin's as well as congruous magnetic behavior with that of the natural molecule displaying the anomaly. In addition, it must, obviously, exhibit similar anomalous behavior in its Mössbauer spectrum. Here, we report on the first requirement.

\subsection{Ferritin}

Ferritin is an ubiquitous protein, widespread among plants, animals, and in several bacteria, that is designed to store and maintain iron in an available, non-toxic form [11-13]. In every case, the molecule consists of a hydrous ferric oxide core sequestered in a roughly spheroidal, $120 \AA$ diameter protein shell. The protein shell, called 'apoferritin', is composed of 24 nearly identical sub-units of molecular weight $\sim 20,000$ daltons, which are arranged to isolate the iron containing core from the cellular environment. Six hydrophilic and hydrophobic channels provide access to the protein interior, presumably for electrons, protons, and iron ions, as well as other small ions.

The ferritin iron core is a hydrous ferric oxide phosphate with nominal formula $(\mathrm{FeOOH})_{8}(\mathrm{Fe}-$ $\mathrm{OH}_{2} \mathrm{PO}_{4}$ ), a structure similar to that of the polycrystalline mineral ferrihydrite. It contains $\mathrm{Fe}^{3+}$ ions octahedrally coordinated to oxygen, i.e. sixfold oxygen coordination, in a crystalline array and oxygens are hexagonally close packed [14]. Phosphate occurs in disordered regions of the core, possibly at the chain ends of the iron polymer and/or at the junction of crystallites with each other or with the protein surface. The core can store up to a maximum of 4500 iron atoms (ions) [15]. When saturated with iron, the core has a diameter of about $80 \AA$ [15] which is the protein shell's inner cavity dimension. The entire ferritin molecule has a molecular weight of about 700,000 daltons (a.m.u.).

\subsection{Polysaccharide iron complex (PIC)}

PIC is a synthetic complex of ferric iron and carbohydrate marketed under the name 'Niferex' as an oral hematinic by Central Pharmaceuticals, Inc. (Seymour, Indiana). It is reported to be effective in the treatment of iron deficiency anemia [16]. PIC is reported to be spheroidal in shape with a $48 \%$ iron content, and having a distribu- 
tion of core volumes with an average diameter of $70 \AA[10,17]$. PIC is synthesized on an industrial scale by Central Pharmaceuticals essentially by a patented procedure [18] which is described in detail elsewhere [1]. It has been suggested [1] that the synthetic PIC molecule resembles in some ways ferritin [19] as well as other iron-carbohydrate complexes such as Imferon [20-22], which contain different forms and concentrations of iron oxyhydroxides. The differences among these iron oxyhydroxides are mainly due to long-range order. Berg [1] has presented evidence from Mössbauer spectroscopy and X-ray diffraction that the iron core in PIC is similar to ferrhydrite and ferritin and has no characteristics of $\beta$-Fe$\mathrm{OOH}$. Hence, PIC apparently differs from Imferon (which contains $\beta-\mathrm{FeOOH}$ ) and is a more suitable model compound ('biomimic') for ferritin, which does not contain $\beta-F e O O H$. Finally, because PIC is partially carbohydrate, the carbohydrate is expected to be external to the iron core. Natural ferritin, however, contains phosphate in its iron core [13], yet has X-ray diffraction and Mössbauer spectra characteristics identical to those of ferritin constituted in the absence of phosphate. These techniques, therefore, may not be able to distinguish between carbohydrate bound on the surface and carbohydrate included in the core.

\section{Experiment}

\subsection{Materials}

PIC was obtained unaltered from Central Pharmaceuticals, Inc. The ferritin used was horse spleen ferritin obtained from the Sigma Chemical Company (St. Louis, MO) and prepared by G.D. Watt of Brigham Young University, Provo, Utah. The method of preparation, described elsewhere [23], produced two types of samples that we shall call Ferritin I (Fer I) and Ferritin II (Fer II). Both samples have an average number of iron ions in their cores equal to 2016 ions [23]. However, for Ferritin II, 1876 ions of the total are $\mathrm{Fe}^{3+}$ while 140 ions are $\mathrm{Fe}^{2+}$, bound mostly to the surface of the core as indicated by Mössbauer experiments [23]. As for Ferritin I, all the iron ions are $\mathrm{Fe}^{3+}$. For PIC, no such detailed estimation of the number of $\mathrm{Fe}^{3+}$ ions in its core has been made. However, given that the average core diameter of PIC is $70 \AA[10,17]$, and knowing the density of ferrihydrite $[13,24]$ as well as its unit cell dimensions, this implies a theoretical number of 5100 iron atoms in the PIC core. Diamagnetic susceptibilities were evaluated for all samples and sample holders and were corrected for.

\subsection{Apparatus}

Experimental measurements were carried out at the Francis Bitter National Magnet Laboratory at MIT (Cambridge, MA), using the SHE variable temperature squid susceptometer (SHE Corp., San Diego, CA) which has a temperature range $2-400 \mathrm{~K}$ and can produce magnetic fields up to $50 \mathrm{kOe}$.

\section{Results}

\subsection{Determination of regions of superparamag- netism}

Both the PIC and ferritin samples are comprised of a distribution of particle sizes $[10,19]$. Experimentally, it is very difficult, but not impossible, to prepare an assemblage of particles with a narrow size distribution, hence, each sample has a distribution of blocking temperatures. Consequently, an important number to determine experimentally is the largest significant blocking temperature in each distribution. To obtain these blocking temperatures, we will determine the region of superparamagnetism for PIC and for Ferritin Types I and II. The method used to determine these regions utilizes the dependence of the coercivity and remanance of an assembly of single-domain superparamagnetic particles on their volume. Néel [25-27] and Brown [28-30] developed theories to deal with such particles.

For an anisotropy having uniaxial symmetry, the free enthalpy or anisotropy energy of such a uniaxial single-domain particle with volume $V$ 
and a magnetization $M_{\mathrm{s}}$ per unit volume, in a field $H$ is given by

$$
E=K V \sin ^{2} \theta-V M_{\mathrm{s}} H \cos (\phi-\theta),
$$

where $\theta$ is the angle between the magnetic moment and the symmetry (easy) axis of the particle, which is considered here for simplicity a prolate spheroid, and $\phi$ is the angle between the field $H$ and the symmetry axis of the particle, and hence, $\phi-\theta$ is the angle between the magnetic moment and the field. In equilibrium, the magnetization $\boldsymbol{M}_{\mathrm{s}}$ assumes a direction $\theta$ (i.e. has an angle $\phi-\theta$ with $H$ ) for which $E$ is a minimum. In sufficiently small fields, $\boldsymbol{M}_{\mathrm{s}}$ has two equilibrium positions, $\theta_{1}$ and $\theta_{2}$, which are separated by an energy barrier.

Néel pointed out that if a single-domain particle were small enough, thermal fluctuations could cause its direction of magnetization to undergo a sort of Brownian motion. He considered a system of $n$ such identical noninteracting single-domain particles and assumed that the particle axes are all aligned in the field direction $(\phi=0)$ for simplicity, and that the particles are in a field $H<$ $H_{\mathrm{K}}=$ anisotropy field of the particle. This is the field at which the energy barrier of the particle vanishes. Néel further assumed that the magnetization of each particle has two equilibrium positions, $\theta_{1}=0$ and $\theta_{2}=\pi$, where initially, $n_{1}$ of the $n$ particles are magnetized along $\theta_{1}$ and the rest, $n_{2}=n-n_{1}$ along $\theta_{2}$ (where this distribution cannot change at temperature $T=0$ ). Calculating [25-27] the barrier height for this assembly gives

$$
\begin{aligned}
\Delta E & =E_{\text {barrier }}=E_{\max }(\theta)-E(\pi) \\
& =K V\left(1+h^{2}\right)-K V(2 h)=K V(1-h)^{2},
\end{aligned}
$$

which gives $H_{\mathrm{K}}=2 K / M_{\mathrm{s}}$, i.e. $h=M_{\mathrm{s}} H / 2 K=1$. According to Néel, fluctuations of $\boldsymbol{M}_{\mathrm{s}}$ about the equilibrium directions are produced thermally. Hence, there will be a finite probability $P_{i j} \mathrm{~d} t$ for $M_{\mathrm{s}}$ of any one of the particles to jump within the time interval $\mathrm{d} t$ from $\theta_{i}$ to $\theta_{j}$.

For the transition probability $P_{i j}$, most theories [52] lead to an expression of the form:

$P_{i j}=f_{i j} \exp \left[-\Delta E_{i} / K_{\mathrm{B}} T\right]$,

where $f_{i j}$ is a frequency factor which has been assumed to slowly vary with temperature.
The magnetic behavior of a fine particle assembly depends on the ratio of the time of the experiment $t_{\mathrm{ex}}$ to the relaxation time $\tau_{0}$. For $t_{\mathrm{ex}} / \tau_{0} \gg 1$, the particle assembly behaves like a paramagnetic gas of 'giant molecules' with moments $V M_{\mathrm{s}}$. The condition for this superparamagnetic behavior $[30,31-36]$ is:

$$
\begin{aligned}
\ln \left(2 t_{\mathrm{ex}} f_{0}\right) \frac{K_{\mathrm{B}} T}{K V} & =\ln \left(2 t_{\mathrm{ex}} f_{0}\right) \frac{2 K_{\mathrm{B}} T}{V M_{\mathrm{s}} H_{K}} \\
& =\ln \left(2 t_{\mathrm{ex}} f_{0}\right) / \nu H_{K}>1 .
\end{aligned}
$$

The volume, $V_{c}$, is the critical particle volume for superparamagnetic behavior:

$V_{\mathrm{c}}=\ln \left(2 t_{\mathrm{ex}} f_{0}\right) \frac{K_{\mathrm{B}} T}{K}=\ln \left(2 t_{\mathrm{ex}} f_{0}\right) \frac{2 K_{\mathrm{B}} T}{M_{\mathrm{s}} H_{K}}$.

For given $T, V>V_{\mathrm{c}}$ blocks superparamagnetism. We must also note that a rough measure of the time used to characterize the transition to stable equilibrium is the blocking temperature, $T_{\mathrm{B}}$, which is the temperature at which the relaxation time becomes of the order of the duration time of the experiment, $t_{\mathrm{ex}}$ :

$T_{\mathrm{B}}=\frac{K V}{\ln \left(2 t_{\mathrm{ex}} f_{0}\right) K_{\mathrm{B}}}$.

In the case of an applied field $H<H_{\mathrm{K}}$, we have (from Eq. (2))

$T_{\mathrm{B}}=\frac{K V(1 \pm h)^{2}}{\ln \left(2 t_{\mathrm{ex}} f_{0}\right) K_{\mathrm{B}}}$,

where \pm depends on whether the field is applied parallel or antiparallel to the magnetization.

The coercivity $H_{\mathrm{c}}$ is that field which reduces the magnetization $\bar{M}(t)$ to zero within the given time $t_{\mathrm{ex}}$ of measurement. When $H$ is large enough to reduce the energy barrier, $\Delta E=K V(1-h)^{2}$ to approximately $25 K T$, the reversal process can be thermally activated within the time of the experiment, $t_{\mathrm{ex}} \approx 10 \mathrm{~s}$. This criterion gives

$H_{\mathrm{c}}=\frac{2 K V}{m}\left[1-5\left(\frac{K_{\mathrm{B}} T}{K V}\right)^{1 / 2}\right]$,

where $H_{\mathrm{K}}=2 K / M_{\mathrm{s}}$ and $m=$ magnetic moment 
of the particle of volume $V ; m=V M_{\mathrm{s}}$. If $V_{\mathrm{c}}$ is introduced by means of Eq. (5), we get

$H_{\mathrm{c}}=\frac{2 K V}{m}\left[1-\left(\frac{V_{\mathrm{c}}}{V}\right)^{1 / 2}\right], \quad V \geq V_{\mathrm{c}}$,

and if $T_{B}$ is introduced by means of Eq. (6b) we have

$H_{\mathrm{c}}=\frac{2 K V}{m}\left[1-\left(\frac{T}{T_{\mathrm{B}}}\right)^{1 / 2}\right], \quad T \leq T_{\mathrm{B}}$.

In the presence of a magnetic field, the energy barrier is less than $K V$ (see Eq. (2)), and therefore the magnetization reversal process can be thermally activated at a lower temperature. From the above, we see that since the time of the experiment is of the order $t_{\mathrm{ex}} \approx 10 \mathrm{~s}$, Eqs. (7), (8) and (9) for the coercivity $H_{c}$, which hold for whatever reversal process the particles find easiest, will describe the coercive field, for the assembly of superparamagnetic particles considered, from $T=0$, where $H_{\mathrm{c}}=2 \mathrm{KV} / \mathrm{m}$, up to $T=T_{\mathrm{B}}$, where $H_{\mathrm{c}}=0$. If $H_{\mathrm{c}}$ is plotted as a function of the square root of the temperature, then the region of superparamagnetism with respect to the applied field $H$ and temperature for a given assembly of particles is that to the right of the plotted curve which intercepts the $H_{\mathrm{c}}$ and $T^{1 / 2}$ axes. Since $V / m$ for the same distribution of the particle volumes is a constant, the value of $H_{c}$ at very low temperatures is independent of the particle size. However, the blocking temperature is directly proportional to the particle volume for a given distribution of particle sizes.

In order to determine values of $H_{\mathrm{c}}$ as a function of temperature for a given sample of superparamagnetic particles, the magnetic measurements were performed in the following manner. All samples were zero field-cooled (ZFC) from above their respective blocking temperatures, determined from Mössbauer spectroscopy, $T_{\mathrm{B}}$ (PIC) $\sim 70 \mathrm{~K}$ [10], $T_{\mathrm{B}}$ (Type II Ferritin) $\sim 58 \mathrm{~K}$ [23], and $T_{\mathrm{B}}$ (Type I Ferritin) $\sim 39 \mathrm{~K}$ [23], down to a temperature of $4.2 \mathrm{~K}$. The magnetic field was turned on and set at a known value. The magnetic moment of the sample was measured as a function of temperature starting at the lowest temperature. The coercive force prevents the magnetization from reaching its new isomagnetic equilibrium value until the sample temperature is brought above its blocking temperature for this magnetic field value. This gives a reproducible, but not reversible magnetization curve. Next, the sample's temperature was first raised well beyond its $T_{\mathrm{B}}$ for the set value of the field. We then lowered the temperature of the sample in the field, i.e. field cooled (FC) the sample while continuously measuring its magnetic moment, down to $4.2 \mathrm{~K}$. This magnetization curve was reproducible and reversible. Both the FC and ZFC magnetization curves superimposed at high temperatures, but bifurcated at a specific temperature. At that intercept of both curves, $H_{\mathrm{c}}=H$, i.e. the applied field is the coercive field $H_{\mathrm{c}}(T)$ at the temperature of the bifurcation point. This was repeated for several field values for the three samples (see Fig. 1).

The general feature of these magnetization curves $m(T)$ are that the specific temperatures at the bifurcation point decreases with increasing field value until the bifurcation is unobservable. The ZFC magnetization curves exhibit a concavity towards the temperature axis at low temperatures below the bifurcation point. The concavity ranges from not at all to pronounced among the different samples at different field values.

Since we are dealing with a distribution of particle volumes, from Eqs. (8) and (9) there exists a corresponding distribution of blocking temperatures and coercivities. Hence for the ZFC curves at a given temperature, there will still be a fraction of the sample with particle volumes that has attained the critical particle volume for superparamagnetic behavior Eq. (5) at that temperature. For such particle volumes, $H_{c}=0$ and the particles are 'unfrozen' and free to rotate so as to achieve their saturation magnetization in that field. However, this is a small fraction of the sample that is behaving this way. As the temperature is raised, a greater fraction of the sample will experience the same phenomena. Therefore, the low-temperature section of the ZFC magnetization curve is in essence a sum of many magnetization curves; hence the concavity. The maximum point of the concave section of the ZFC curve 

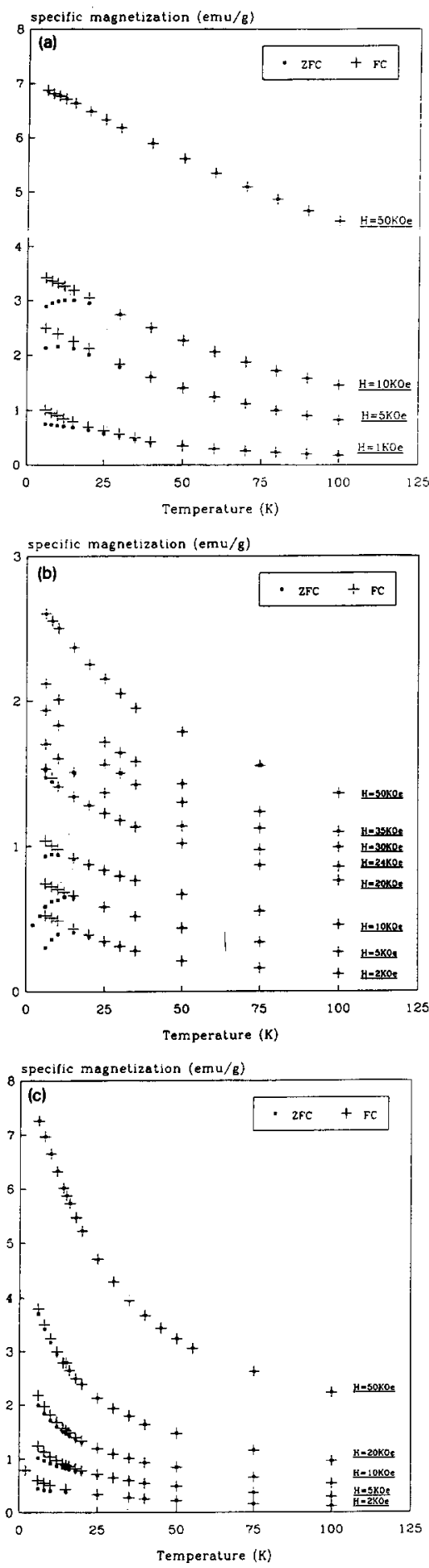

Fig. 1. Specific magnetization $m(T)(\mathrm{emu} / \mathrm{g})$ at constant field $H$ (kOe) for: (a) PIC; (b) Ferritin I; (c) Ferritin II. ZFC-zero field cooled, $\mathrm{FC}=$ field cooled. therefore corresponds to the blocking temperature of the average particle volume in the distribution, and the bifurcation point, where the ZFC curve joins the FC curve, corresponds to the blocking temperature of the largest particle volume. Thus the entire sample is 'unblocked' at this field and temperature.

For larger field values the barrier height will decrease further, i.e. $h$ in $\mathrm{Eq}$. (6b) will increase and will result in lower blocking temperatures for all particle volumes; hence the shift to lower temperatures corresponding to the bifurcation point and the maximum of the concave sections. When the blocking temperature corresponding to the average particle volume becomes unobservably low, the maximum in the ZFC disappears.

A plot of $H_{c}$ as a function of $T^{1 / 2}$ for PIC, Type II $\left(\mathrm{Fe}^{2+}+\mathrm{Fe}^{3+}\right)$ and Type $\mathrm{I}\left(\mathrm{Fe}^{3+}\right)$ ferritin is shown in Fig. 2. A fit of the coercive force Eq. (9) yields the following values:

$2 \mathrm{KV} / \mathrm{m}=36 \mathrm{kOe}$ and maximum $T_{\mathrm{B}}=48 \mathrm{~K}$ at $H=0$ for PIC;

$2 \mathrm{KV} / \mathrm{m}=38 \mathrm{kOe}$ and maximum $T_{\mathrm{B}}=29 \mathrm{~K}$ at $H=0$ for Type I $\left(\mathrm{Fe}^{3+}\right)$ ferritin;

$2 \mathrm{KV} / \mathrm{m}=43 \mathrm{kOe}$ and maximum $T_{\mathrm{B}}=39 \mathrm{~K}$ at $H=0$ for Type II $\left(\mathrm{Fe}^{2+}+\mathrm{Fe}^{3+}\right)$ ferritin.

This plot defines the region where each sample behaves like a superparamagnet. For example, for PIC it is superparamagnetic above $48 \mathrm{~K}$ at $H=0$, whereas at $H=10 \mathrm{KOe}$ the temperature above which it is superparamagnetic has been reduced to $20 \mathrm{~K}$. In the region to the left of the curves in Fig. 2, $H<H_{\mathrm{c}}(T)$, the sample is not superparamagnetic.

This experimental method to determine $H_{\mathrm{c}}$ is quite accurate. This is because in conventional methods of measuring $H_{\mathrm{c}}$ one obtains a value for the total coercive force for all particles, whereas in this method one obtains $H_{\mathrm{c}}$ for only that fraction of the particles with the largest product $K V$. For PIC the average particle diameter $d$ has been estimated from Mössbauer measurements and the distribution of hyperfine fields to be equal to $70 \AA[10,17]$. This value corresponds to a volume of $1.8 \times 10^{-19} \mathrm{~cm}^{3}$. Also, the anisotropy constant for PIC was estimated from Mössbauer data $[10,17]$ to be $K=3.0 \times 10^{5} \mathrm{erg} / \mathrm{cm}^{3}$. Hence, from the previous value obtained from $H_{\mathrm{c}}(T=0)$ $=2 \mathrm{KV} / \mathrm{m}$ we estimate the magnetic moment of 

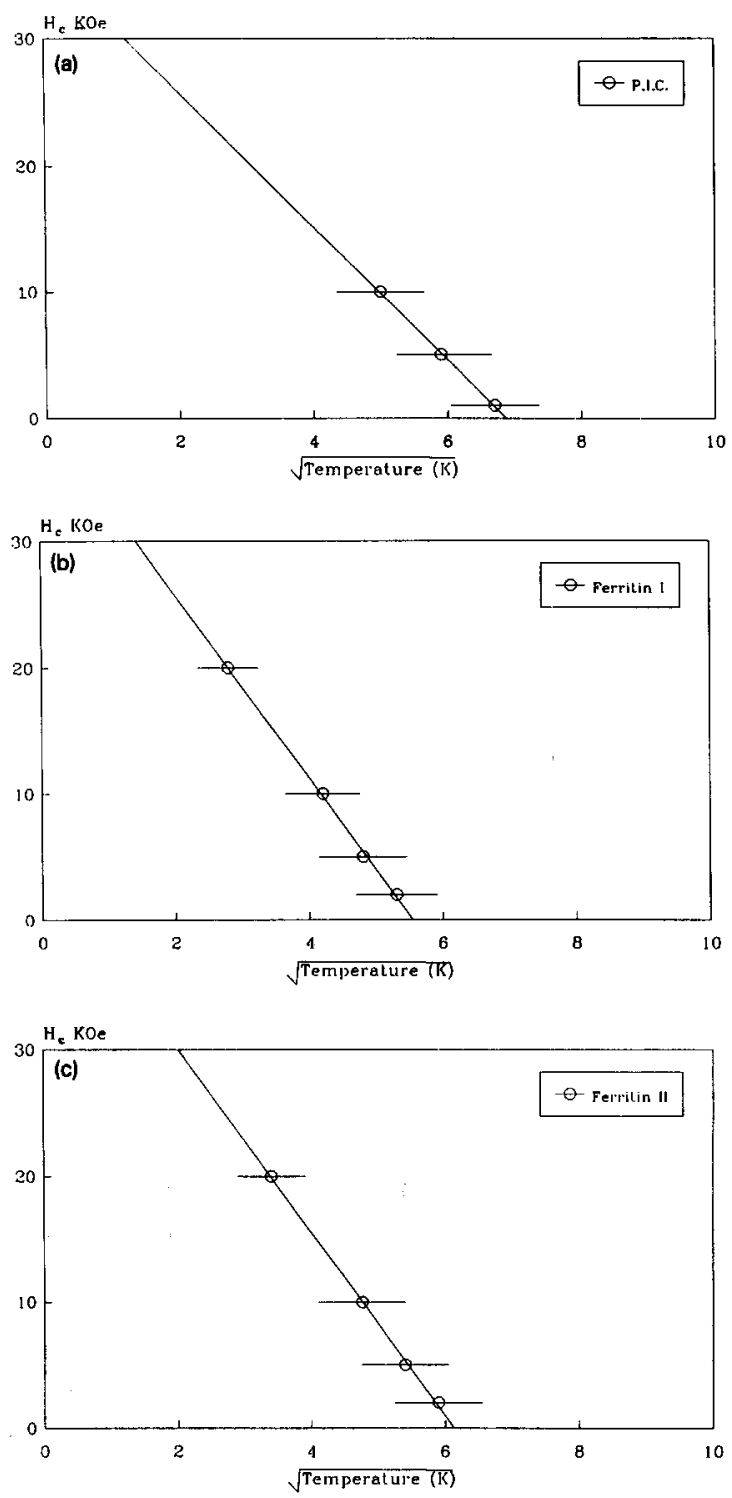

Fig. 2. Coercive force $H_{\mathrm{c}}(\mathrm{kOe})$ versus the square root of the temperature $T^{1 / 2}\left(\mathrm{~K}^{1 / 2}\right)$ for: (a) PIC; (b) Ferritin I; (c) Ferritin II. The solid line is a fit to Eq. (9).

the average PIC particle to be $m_{\mathrm{PIC}}=301 \mu_{\mathrm{B}}$ (Bohr magneton). As for Ferritin Type I $\left(\mathrm{Fe}^{3+}\right)$, the particle size distribution has been determined for similar mammalian ferritin containing a range of $\mathrm{Fe}^{3+}$ ions from 1730 to 2480 [19]. The average diameter was estimated to be $62 \AA$, which gives a volume of $1.3 \times 10^{-19} \mathrm{~cm}^{3}$. The anisotropy con- stant for ferritin is assumed by many researchers [37] to be of the order $K=10^{4} \mathrm{erg} / \mathrm{cm}^{3}$, which is that for $\mathrm{FeOOH}$ [38]. However, St. Pierre and others $[8,9,19]$ showed that it should at least equal $K=10^{5} \mathrm{erg} / \mathrm{cm}^{3}$, if not higher. Hence, using these values with the $H_{\mathrm{c}}(T=0)$ for Ferritin Type I to get an order of magnitude for the magnetic moment of the ferritin molecule, we get $m_{\text {FerI }} \sim 100 \mu_{\mathrm{B}}$. More information on the magnetic behavior of the Ferritin I sample is needed to obtain a more accurate value.

\subsection{Field magnetization measurements}

Magnetization measurements were also carried out as a function of the magnetic field at various values of constant temperatures. Fig. 3 shows the magnetization curves obtained in this manner. The curves are linear for low fields, curving downwards for higher fields. The crossover field between the two regions increases with decreasing values of temperature, for other mammalian ferritin [6] (with no $\mathrm{Fe}^{2+}$ ions added) the magnetic field value at which the tendency to linearity begins in the magnetization curve is about $10 \mathrm{kOe}$ for temperatures lower than $T=20$ $K$. On the other hand, for high temperatures ( $T \geq 200 \mathrm{~K})$, the concavity does not exist and the curves are linear throughout the measured values of field. The other important feature of these magnetization curves is that they do not saturate up to $50 \mathrm{kOe}$.

These features of the magnetization curves reflect a combination of two types of superimposed magnetic phenomena, each with a different temperature-dependent magnetization. These are the superparamagnetism of the uncompensated spins of the particles and the bulk antiferromagnetism of the particle cores. In fact, for a given magnetic field value, the magnetization is composed of two terms, one is a superparamagnetic magnetization with a saturation magnetization of $M_{\mathrm{s}}$, the other an antiferromagnetic magnetization that is proportional to the magnetic field.

We divide the magnetization curves into four regions: (1) low magnetic fields $(H \leq 5 \mathrm{kOe})$ and $T<T_{\mathrm{B}}$, (2) low magnetic fields $(H \leq 5 \mathrm{kOe})$ and 

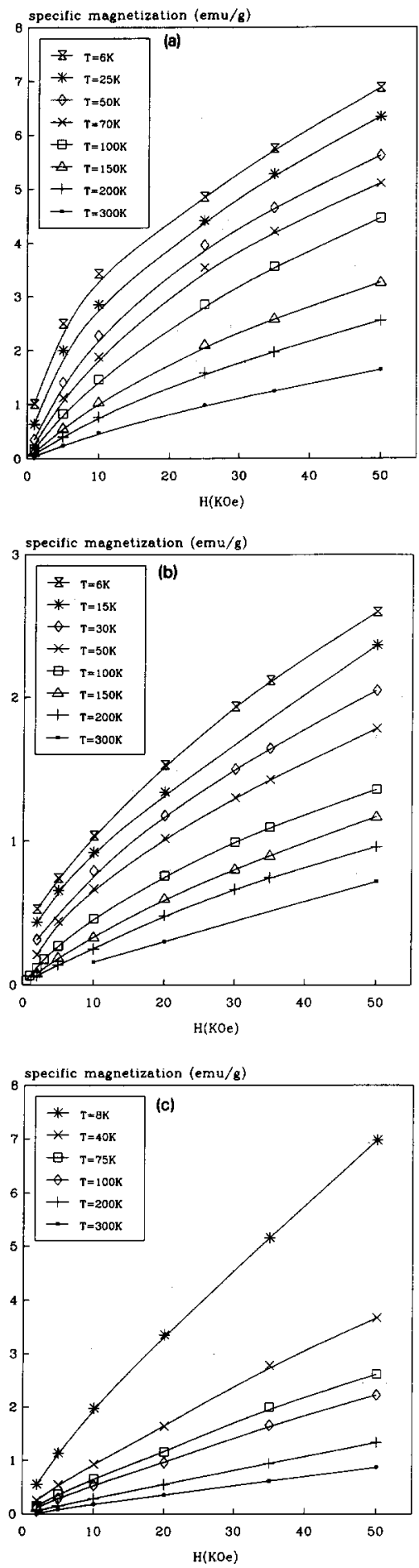

Fig. 3. Specific magnetization $m(H)(\mathrm{emu} / \mathrm{g})$ at constant temperature $T$ (K) for: (a) PIC; (b) Ferritin I; (c) Ferritin II. Solid lines through the experimental points are a graphical aide.
$T>T_{\mathrm{B}}$, (3) high magnetic fields and $T<T_{\mathrm{B}}$, and (4) high magnetic fields and $T>T_{\mathrm{B}}$.

Region (1): $H \leq 5 \mathrm{kOe}, T<T_{\mathrm{B}}$. In this region there exist three contributions to the magnetization. The first is a superantiferromagnetic magnetization contribution. The second is a superparamagnetic magnetization contribution and the third is an antiferromagnetic magnetization contribution. The first contribution exists in this region because particles with the largest volumes contribute significantly to the low field magnetization process, whereas the smaller particles do not. The larger particles are those that produce the superantiferromagnetic effect below their blocking temperatures (Néel [39]), while below the blocking temperatures of these large particles, the smaller ones are still superparamagnetic and make the second contribution to the magnetization in this region. As for the last contribution, it is in general smaller than the other two, given the relatively temperature-independent (field-proportional) antiferromagnetic magnetization of comparable 'bulk' material at such low fields. We note that the dominant order of these contributions will vary with temperature in this region up to $T_{\mathrm{B}}$.

Region (2): $H \leq 5 \mathrm{kOe}, T>T_{\mathrm{B}}$. In this region, the magnetization will be a simple superposition of the two contributions, the superparamagnetic and the antiferromagnetic. This is valid according to Néel [40] as long as the thermal energy $K_{\mathrm{B}} T$ exceeds both magnetic energies. Near the blocking temperature, the superparamagnetic magnetization will be dominant and will continue to be so up to $T \sim 100 \mathrm{~K}$ for Ferritin II, $T \sim 150 \mathrm{~K}$ for Ferritin I and PIC. In large part, this may be traced to the Curie law $1 / T$ dependence of the superparamagnetic magnetization compared to the weak temperature dependence, at these field values, of the antiferromagnetic magnetization.

Region (3): high magnetic field ( $>20 \mathrm{kOe}$ ), $T<T_{\mathrm{B}}$. The contributions to the magnetization in this region are the same as in region (1), however, the order of dominance is different, since here the superparamagnetism magnetization saturates. From the regions of the super- 
paramagnetism for ferritin and PIC (Fig. 2), we see that the total sample becomes superparamagnetic at temperatures much lower than their maximum blocking temperature $T_{\mathrm{B}}$ for higher fields. Hence, saturation of the superparamagnetism magnetization will occur at still higher fields and coincide with the appearance of the tendency to linearity in conjunction with the unsaturated growth of the magnetization curves. Unfortunately, the truly linear section of the magnetization curve in this region will occur at very high field values, where the magnetization will still continue to grow due to the two other contributions.

Region (4): high magnetic field ( $>20 \mathrm{kOe}$ ), $T>T_{\mathrm{B}}$. The difference between this region and region (2) is that, with higher temperatures, the superparamagnetic magnetization contribution will saturate at lower field values than it does in region (3), and will produce similar asymptotic behavior in the concave magnetization curves. In
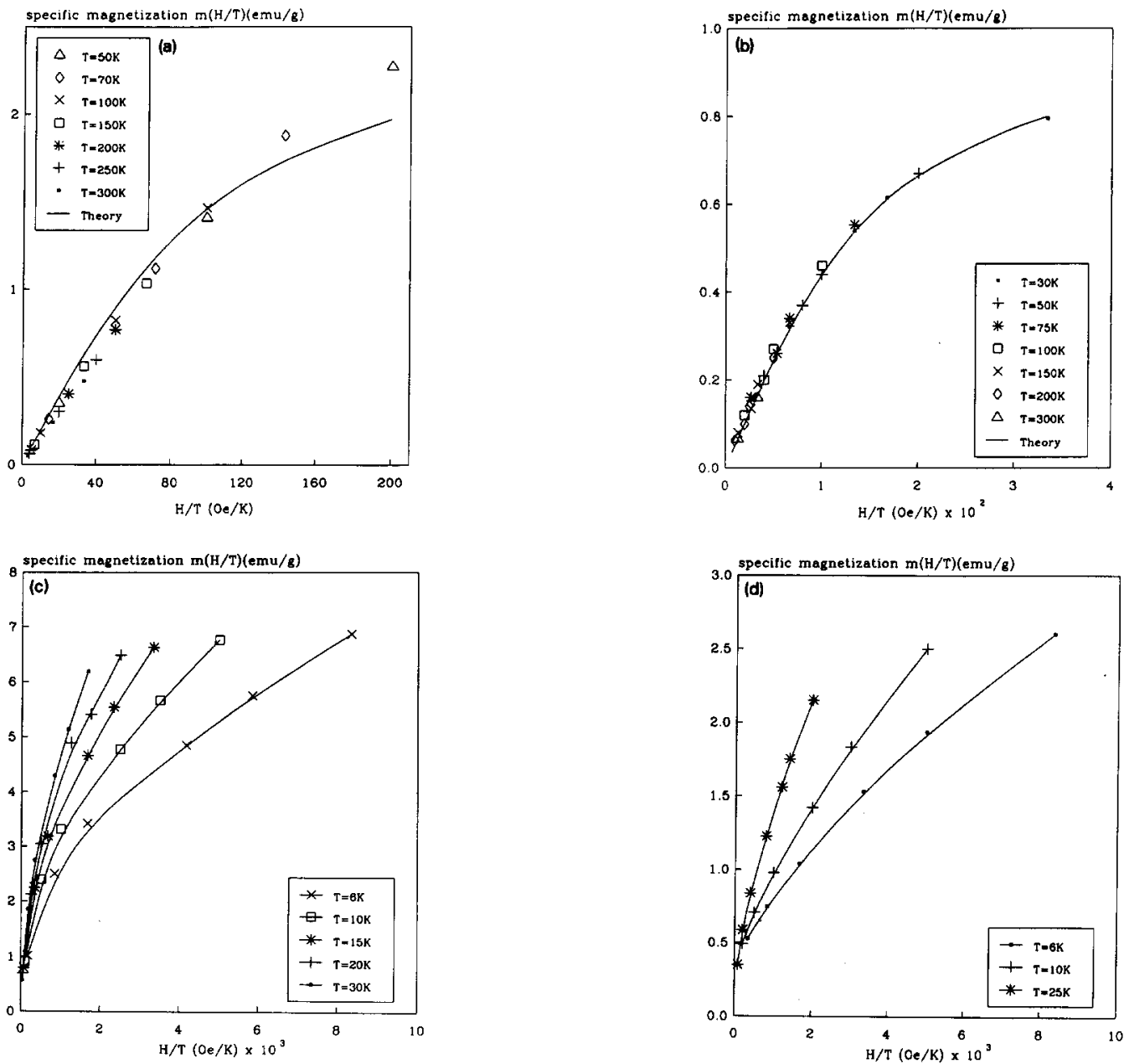

Fig. 4. Specific magnetization $m(H)$ (emu/g) versus $H / T$ (kOe/K) for: (a) PIC for $T>T_{\mathrm{B}}=48 \mathrm{~K}$; solid line represents the fit to a Langevin function which yields a saturation magnetization $m_{\mathrm{s}}=2.6 \mathrm{emu} / \mathrm{g}$ and a magnetic moment $m=310 \mu_{\mathrm{B}}$. (b) Ferritin I, for $T>T_{\mathrm{B}}=29 \mathrm{~K}$; solid line represents the fit to a Langevin function which yields a saturation magnetization $M_{\mathrm{s}}=1 \mathrm{emu} / \mathrm{g}$ and a magnetic moment $m=200 \mu_{\mathrm{B}}$. (c) PIC, for $T<T_{\mathrm{B}}$. Solid lines through the experimental points are graphical aides. (d) Ferritin I, for $T<T_{\mathrm{B}}$. Solid lines through the experimental points are graphical aides. 
the asymptote or linear section of the curve, the magnetization's growth will only be due to the remaining antiferromagnetic contribution. In this region, the intersection of the linear section of the magnetization curve with the magnetization axis, particularly for those curves at temperatures ( $T \geq T_{\mathrm{B}}$ ) at or close to the blocking temperature, can give an experimentally more accurate estimation of the superparamagnetic saturation magnetization $M_{\mathrm{s}}$, than it does in region (3), especially if one is working with moderately high (up to 50 $\mathrm{kOe})$ and not extremely high (500 kOe) magnetic fields. From this region of the magnetization curves we determined for PIC $M_{\mathrm{s}}=2.5 \mathrm{emu} / \mathrm{g}$, for Ferritin I $M_{\mathrm{s}}=0.93 \mathrm{emu} / \mathrm{g}$, and for Ferritin II $M_{\mathrm{s}}=1.2 \mathrm{emu} / \mathrm{g}$.

One must take all effects into consideration in deriving quantitative information from PIC and ferritin's magnetization curves. To do so we concentrate on region (2) of the curves where superparamagnetism is dominant. Using the second part of the operational definition of superparamagnetism which is the superposition principle [41]: the magnetization curve, barring particle interactions, for a relatively isotropic sample, must be temperature-dependent to the extent that curves taken at different temperatures must approximately superimpose when plotted against $H / T$. In Figs. 4(a) and (b), the magnetization of PIC and Ferritin I from region (2) (up to $5 \mathrm{kOe}$ ) is plotted as a function of $H / T$. While in Figs. 4(b) and (c), the corresponding magnetizations from regions (1) and (3) are also plotted similarly. One notices immediately that there is no superposition of the curves in the second set of figures where the temperature is lower than the blocking temperature in these regions. However, the superposition of the curves is evident in the first set of figures where the superparamagnetism is the dominant magnetization in that region. It is also evident from the superposed curves that there is no need to apply the correction of Abeledo and Selwood [42] for the temperature dependence of the spontaneous magnetization $M_{\mathrm{s}}$. They assumed that the spontaneous magnetization per unit volume $M_{\mathrm{s}}(T)(=m / V)$ at temperature $T$, is independent of $V$ and that if a sample of super- paramagnetic particles has an anomalous temperature dependence of its spontaneous magnetization $M_{\mathrm{s}}$, then $M_{\mathrm{s}}$ for such particles does not follow the temperature dependence of the spontaneous magnetization of the particle's bulk material. Hence the magnetization curves of these particles will not superimpose, unless the magnetization is multiplied by the factor $M_{\mathrm{s}}(T=0$ $\mathrm{K}) / M_{\mathrm{s}}(T)$ and plotted against $H M_{\mathrm{s}}(T) / T M_{\mathrm{s}}(0)$, where $M_{s}(0)$ is the spontaneous magnetization per unit volume at $T=0 \mathrm{~K}$. Therefore, the superposition of the magnetization curves for PIC and ferritin is evidence for normal behavior of their $M_{\mathrm{s}}(T)$ in this region (2). This is fortunate because otherwise we would have needed to obtain the values of $M_{\mathrm{s}}(T)$ and $M_{\mathrm{s}}(0)$ from the magnetic properties of the bulk state of PIC and ferritin. In the case of ferritin, however, nature gives us only a fine-grained product whose bulk state no one has succeeded in producing so far, in spite of use of very high hydrostatic pressure [5,6].

The magnetization (per unit mass) of an isotropic assembly of $n$ superparamagnetic particles with a distribution $P(V)$ of particle volumes is given by [39]:

$$
\begin{aligned}
m & =V_{\mathrm{T}} M_{\mathrm{s}} \int_{0}^{\infty}\left(\operatorname{coth}\left(\frac{V M_{\mathrm{s}} H}{K_{\mathrm{B}} T}\right)-\frac{K_{\mathrm{B}} T}{V M_{\mathrm{s}} H}\right) P(V) \mathrm{d} V \\
& =\sum_{i}^{n} V_{i} M_{\mathrm{s}}\left(\operatorname{coth}\left(\frac{V_{i} M_{\mathrm{s}} H}{K_{\mathrm{B}} T}\right)-\frac{K_{\mathrm{B}} T}{V_{i} M_{\mathrm{s}} H}\right),
\end{aligned}
$$

where $V_{\mathrm{T}}$ is the volume (per unit mass) of the assembly, $M_{\mathrm{s}}$ is assumed to be independent of particle volume and $m_{\mathrm{s}}=\sum_{i}^{n} V_{i} M_{\mathrm{s}}$ is the superparamagnetic saturation magnetization of such an assembly. If the distribution of the assembly of particles is relatively narrow, Eq. (1) will approximate the regular Langevin function. Mössbauer data for PIC [10] has revealed a distribution of particle volumes with a width $\sigma(d) \sim 25 \AA$. However, we used a regular Langevin function to least square fit the superimposed magnetization curves of PIC (from region (2)). The solid line in Fig. 4(a) shows this fit. The fit yields the following values for PIC: $M_{\mathrm{s}}=2.6 \mathrm{emu} / \mathrm{g}, m=310 \mu_{\mathrm{B}}$, which is in good agreement with $m$ obtained from the determination of the superparamagnetic 
region of PIC and $M_{\mathrm{s}}$ from the intercept of the (asymptote) extension of the linear section of the magnetization curve (for $T \geq T_{\mathrm{B}}$ ) with the magnetization axis (Fig. 3a).

For Ferritin I, a similar fit was done (solid line in Fig. 4b), which yielded the values: $M_{\mathrm{s}}=1$ emu $/ g$ and $m=200 \mu_{B}$. The fit appears to be very good, indicating that the distribution of ferritin particle volumes is narrow (narrower than that for PIC). $M_{\mathrm{s}}$ determined from the fit is in good agreement with that value determined graphically from Fig. 3(b). The value of $m$ is close to the value $217 \mu_{\mathrm{B}}$ obtained recently in experiments by Awschalom et al. [43] on macroscopic quantum tunneling in horse spleen ferritin using an integrated dc SQUID microsusceptometer.

For Ferritin II, a similar fit for the magnetization was done and yielded the same value for $m$ as in Ferritin I, but gave $M_{\mathrm{s}}=1.25 \mathrm{emu} / \mathrm{g}$, which is in good agreement with the value of $M_{\mathrm{s}}=1.2$ emu /g obtained graphically from Fig. 3(c).

\subsection{Magnetic remanance}

The magnetic remanance (thermo-remanent magnetization, TRM) [25-27] of the PIC and Ferritin I samples was also measured as a function of temperature. Both samples were fieldcooled at $50 \mathrm{kOe}$ from a temperature $T=300 \mathrm{~K}$ then to a temperature $T=6 \mathrm{~K}$, and thus below

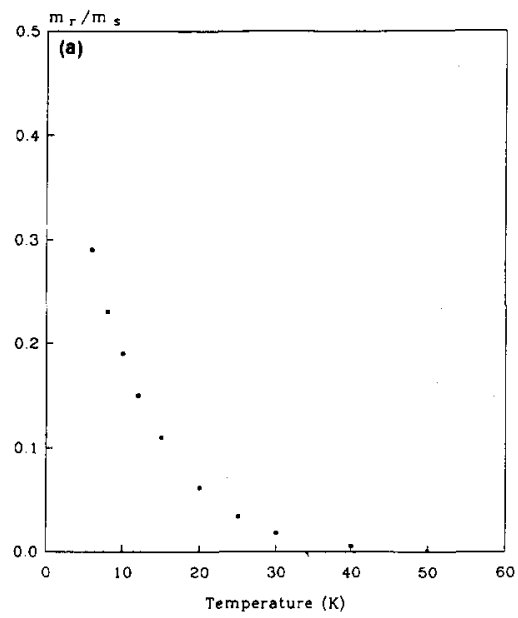

their blocking temperatures. The magnetic field was then switched off, and the magnetic remanance was measured as the temperature was increased. The ratio of remanance to superparamagnetic saturation magnetization $M_{\mathrm{s}}$ (obtained previously) was plotted as a function of temperature (see Fig. 5). Note that the remanance (TRM) magnetization becomes negligible with respect to the saturation magnetization at approximately $T$ $=30 \mathrm{~K}$ for Ferritin I and $T=50 \mathrm{~K}$ for PIC, which are their maximum blocking temperatures as determined from the regions of superparamagnetism (see Fig. 2), i.e. the coercive force $H_{c}$ is zero above those temperatures for the entire sample for Ferritin I and PIC, respectively. For randomly oriented single-domain superparamagnetic particles, assuming the dominant anisotropy is uniaxial [44], the remanance is expected to approach a value of $1 / 2$ the saturation magnetization as $T \rightarrow 0 \mathrm{~K}$ (when the particles are completely stable). Both PIC and ferritin seem to satisfy this condition.

\subsection{Magnetic susceptibilities}

The initial susceptibility was obtained from regions (1) and (2) of the magnetization curves of Figs. 3(a)-(c) for low magnetic fields ( $\leq 5 \mathrm{kOe})$. In Figs. 6(a)-(c) are the plots of the initial 'total' susceptibility and its inverse as a function of the

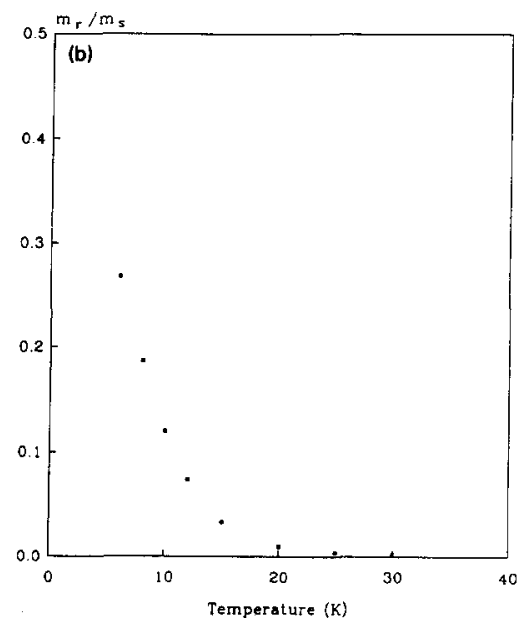

Fig. 5. Remanent (specific) magnetization (TRM) $m_{\mathrm{r}}$ (emu/g). Field cooled in magnetic field $H=50 \mathrm{kOe}$. $H$ switched-off at $T=6$ $\mathrm{K}$ for: (a) PIC; (b) Ferritin I. $m_{\mathrm{s}}=$ saturation magnetization. 

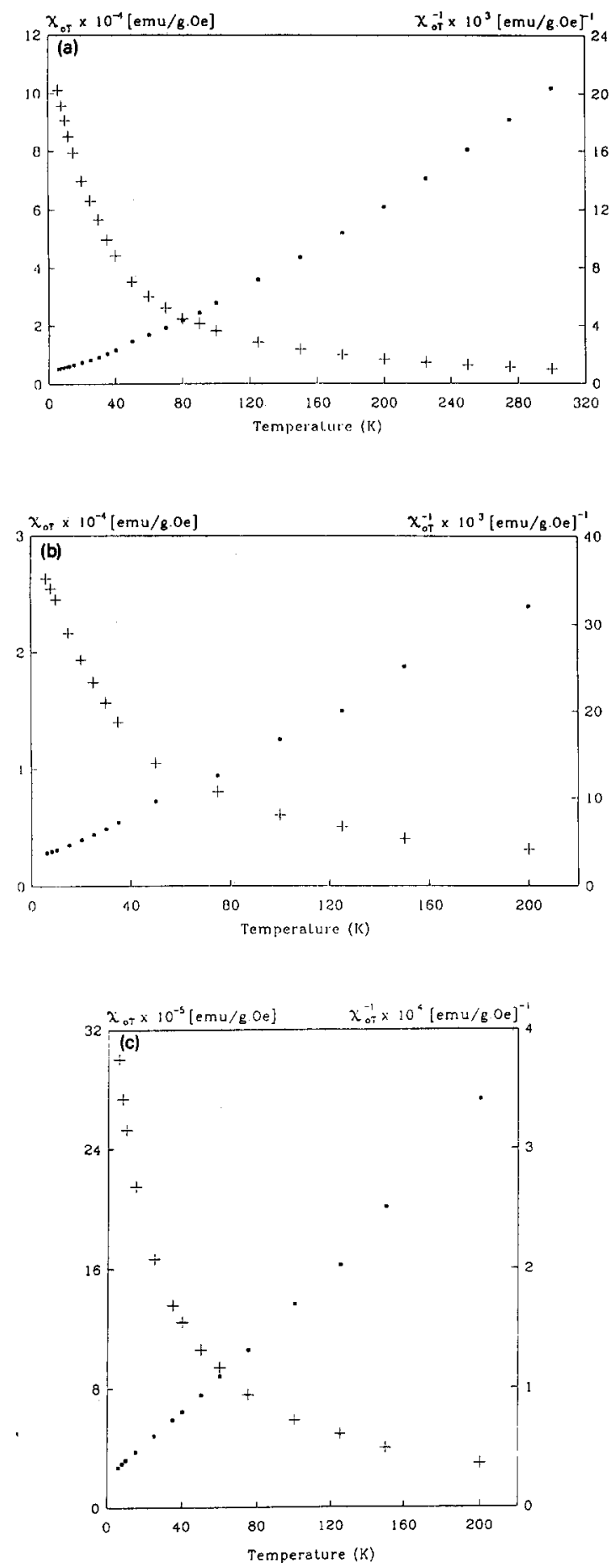

Fig. 6. Total (initial) $\chi_{\mathrm{OT}}(T)$ and inverse $\chi_{\mathrm{OT}}^{-1}(T)$ susceptibilities versus temperature $T$ (K) for: (a) PIC; (b) Ferritin I; (c) Ferritin II. temperature for PIC, Ferritin I, and Ferritin II, respectively.

We have noted that the magnetization is a sum of several contributions in these regions. Hence, the initial susceptibility is a sum of several susceptibilities corresponding to these contributions to the magnetization. This indicates that fitting such a susceptibility curve is not simple. However, extracting information from it is. From the inverse initial susceptibility curve it is evident that there exists an antiferromagnetic interaction. In region (2) $\left(H<5 \mathrm{kOe}, T>T_{\mathrm{B}}\right)$ we noted that the magnetization becomes a simple sum of two contributions as long as the thermal energy exceeds both magnetic energies. Hence the initial 'total' susceptibility $\chi_{0 \mathrm{~T}}$ in this region becomes a simple sum of two susceptibilities; a superparamagnetic $\chi_{\mathrm{p}}$ susceptibility and an antiferromagnetic $\chi_{\mathrm{A}}$ susceptibility,

$\chi_{0 T}=\chi_{\mathbf{P}}+\chi_{\mathrm{A}}$.

In region (4) $\left(H>20 \mathrm{kOe}, T \geq T_{\mathrm{B}}\right)$, the superparamagnetic magnetization will saturate, as we have seen in Section 3.3. Hence, the slopes of the linear sections of the magnetization curve in this region will yield $\chi_{\mathrm{A}}$. In Figs. $7(\mathrm{a})-(\mathrm{c})$ the antiferromagnetic susceptibility $\chi_{\mathrm{A}}$ obtained in this way is plotted, along with the inverse susceptibility $\chi_{\mathrm{A}}^{-1}$ as a function of temperature $T$, where $T_{\mathrm{B}} \leq$ $T \leq 200 \mathrm{~K}$ for PIC, Ferritin I and Ferritin II. All the inverse susceptibilities fit a Curie-Weiss law $\left[\chi_{\mathrm{A}}\right]^{-1}=\left[C_{\mathrm{A}} /(T+\Theta)\right]^{-1}$, where $C_{\mathrm{A}}$ is the Curie constant given by

$C_{\mathrm{A}}=\frac{N_{\mathrm{sg}} \mu_{i}^{2}}{3 K_{\mathrm{B}}}$.

Here, $N_{\mathrm{sg}}=N_{\mathrm{sv}} / \rho=$ number of magnetic moments 'spins' per gram in the sample, $N_{\mathrm{sv}}=$ number of magnetic moments per $\mathrm{cm}^{3}, \rho$ is the sample's mass density, and $\mu_{i}=$ the magnetic moment for each ion. From the values of $N_{\mathrm{sg}}$ for ferritin $\left(1.3 \times 10^{21}\right.$ iron ion $\left./ g\right)$ and PIC $\left(3 \times 10^{21}\right.$ iron ion $/ g$ ) and the values of the slope in Figs. 7(a) and (b) we obtain the magnetic moment per iron ion in Ferritin $\left(\mu_{i}=5.2 \mu_{\mathrm{B}}\right)$ and in PIC $\left(\mu_{i}=4.7 \mu_{\mathrm{B}}\right)$. The theoretical value for the magnetic moment of $\mathrm{Fe}^{3+}$ is $5.92 \mu_{\mathrm{B}}$. Blaise et al. 
[5,6] has reported a value of $\sim 5.08 \mu_{\mathrm{B}}$ for similar mammalian ferritin. The difference in these values may be due to the higher density and order of PIC which may increase the number of antiferromagnetic interactions between the atoms, i.e. the next-nearest interactions become appreciable. On the other hand, the low density may contribute to the higher value for ferritin. From the magnetic moments per particle we estimate the number of ions which produce the particle's magnetic moment or the 'number of uncompensated spins' $n_{\mathrm{u}}$ in each molecule: for PIC $n_{\mathrm{u}}=64$, while $n_{\mathrm{u}}=\mathbf{3 9}$ for ferritin.

We also obtained the following values for the Curie-Weiss $\Theta$ : $\Theta=150 \mathrm{~K}$ for PIC, $\Theta=239 \mathrm{~K}$ for Ferritin I, and $\Theta=160 \mathrm{~K}$ for Ferritin II. For Ferritin $I$, the value of $\Theta$ is in excellent agreement with the accepted Néel ordering temperature of $T_{\mathrm{N}}=240 \mathrm{~K}$, i.e. $\Theta / T_{\mathrm{N}}=1$ since all nextnearest or other neighbors' interactions in Ferritin I are small with respect to the nearestneighbor interaction. However, in Ferritin II, since the only difference with Ferritin $I$ is that $7.5 \%$ of its iron ions are $\mathrm{Fe}^{2+}$ and the rest are $\mathrm{Fe}^{3+}$, it is apparent that one of the effects of the $\mathrm{Fe}^{2+}$ ions is that the next-nearest and other neighbor interactions now contribute to the magnetic behavior of the Ferritin II molecules as well as that this also indicates a rise of the intra-site interaction, i.e. sites on the same sublattice. This reasoning may also apply for PIC since its expected Néel temperature $T_{\mathrm{N}} \sim 200 \mathrm{~K}$, which is that for ferrihydrite, is greater than what we found for $\Theta$. However, the cause for such enhanced interactions in PIC is not the existence of an appreciable amount of $\mathrm{Fe}^{2+}$ ions, rather it is due to the fact that it has a higher degree of order and density than the Ferritin I molecules.

From the initial 'total' and antiferromagnetic susceptibilities obtained from regions (2) and (4), respectively, of the magnetization curves one can extract the $\chi_{\mathrm{p}}$ superparamagnetic susceptibility, and its inverse. The inverse susceptibility for all samples satisfies a Curie law $\left[\chi_{\mathrm{p}}\right]^{-1}=\left[C_{\mathrm{p}} / T\right]^{-1}$, where the Curie constant is

$C_{\mathrm{P}}=\frac{N_{\mathrm{pg}} m^{2}}{3 K_{\mathrm{B}}}$, where $N_{\mathrm{pg}}$ is the number of particles per gram (mass susceptibilities) and $m$ is the magnetic moment per particle.

For a uniaxial anisotropic assembly of superparamagnetic particles [44] with the direction of their symmetry axes aligned with the direction of the magnetic field $H$, the low field fractional magnetization will depend on the value of $K V / K_{\mathrm{B}} T$, and for $K>0$ will vary from $N_{\mathrm{pg}} m^{2} H / 3 K_{\mathrm{B}} T$ for $K V<K_{\mathrm{B}} T$ to $N_{\mathrm{pg}} m^{2} H / K_{\mathrm{B}} T$ for $K V>$; hence the initial susceptibility will vary accordingly, i.e. increasing from $C_{\mathrm{p}} / K_{\mathrm{B}} T$ to $3 C_{\mathrm{p}} / K_{\mathrm{B}} T$ as $K V / K_{\mathrm{B}} T$ increases. If the applied magnetic field is perpendicular to the easy symmetry axis the low field initial susceptibility will decrease with increasing $K V / K_{\mathrm{B}} T$. However, if the directions of the particles symmetry axis is distributed at random, the initial fractional magnetization (susceptibility) of the random assembly remains at $N_{\mathrm{pg}} m^{2} H / 3 K_{\mathrm{B}} T\left(N_{\mathrm{pg}} m^{2} / 3 K_{\mathrm{B}} T\right)$. The dominant term governing the approach to the superparamagnetic saturation magnetization of such particles will still be $K_{\mathrm{B}} T / m H$, regardless of the direction of $H$ and the magnitude of $K V / K_{\mathrm{B}} T$. If instead of a uniaxial anisotropy, the single domain particles had another type of anisotropy, e.g. cubic anisotropy, the $N_{\mathrm{pg}} \mathrm{m}^{2} /$ $3 K_{\mathrm{B}} T$ expression for the initial susceptibility will still be applicable for any direction of applied field and any value of $K V / K_{\mathrm{B}} T$.

Due to the antiferromagnetic structure of the single-domain particles of PIC and ferritin, their magnetic moment will be produced, according to Néel [39], by a number of uncompensated spins $n_{\mathrm{u}}$. If the particles are very fine, with diameters less than $40-50 \AA$, with a large degree of imperfection in its internal structure and surface, then $n_{\mathrm{u}}$ is of the order $N_{\mathrm{sp}}^{1 / 2}$, where $N_{\text {sp }}$ is the number of magnetic ions 'spins' per particle. On the other hand, for larger particles ( $d>50 \AA$ ) with fewer imperfections in their core structures and surfaces, $n_{\mathrm{u}}$ is equal to $N_{\mathrm{sp}}^{2 / 3}$, where now the uncompensated spins will be on the particle's surface. One notes that all such uncompensated spins do not lie or are not aligned in one direction. In fact, if they are ideally and completely randomized then $n_{\mathrm{u}}$ will equal $N_{\mathrm{sp}}^{1 / 4}$ for smaller fine particles and $N_{\mathrm{sp}}{ }^{1 / 3}$ for the larger particles in reality 
one expects to find

$N_{\mathrm{sp}}^{1 / 4}<n_{\mathrm{u}}=N_{\mathrm{sp}}^{x}<N_{\mathrm{sp}}^{1 / 2}$

for very fine particles $(d<20 \AA)$,

$N_{\mathrm{sp}}^{1 / 3}<n_{\mathrm{u}}=N_{\mathrm{sp}}^{x}<N_{\mathrm{sp}}^{2 / 3}$ for larger particles.

Hence the magnetic moment of such particles can be written as $m=N_{\mathrm{sp}}^{x} \mu_{i}$, where $\mu_{i}$ is the magnetic moment per ion. Also, $N_{\mathrm{sp}}, N_{\mathrm{pg}}, N_{\mathrm{sg}}$ as defined above fulfill the relation, $N_{\mathrm{sg}}=N_{\mathrm{pg}} N_{\mathrm{sp}}$. Hence, from the values of the magnetic moment per particle for Ferritin I and PIC we get $N_{\mathrm{pg}}=$ $8.22 \times 10^{17} \mathrm{PIC}$ particles $/ \mathrm{g}$ and $N_{\mathrm{pg}}=6.3 \times 10^{17}$ Ferritin I particles/g. This yields $N_{\mathrm{sp}}=2063$ for ferritin which is in good agreement with the actual average $N_{\mathrm{sp}}=2016$ that has already been determined for Ferritin I. For PIC we get $N_{\mathrm{sp}}=$ 3752. From the uncompensated spins (magnetic moments) determined earlier we have for Ferritin I: $(2016)^{x}=39$ which gives $x=0.481$, while for PIC: $(3752)^{x}=64$ yields $x=0.509$. Blaise [6] has obtained $n_{\mathrm{u}}=26$ and $N_{\mathrm{sp}}=730$ for similar mammalian ferritin, which also satisfy the relation in Eq. (14b) as do our samples.

We indicated earlier that the initial susceptibility $\chi_{0 \mathrm{~T}}$ should be a sum of the antiferromagnetic and superparamagnetic susceptibilities in region (2) $\left(H<5 \mathrm{kOe}, T>T_{\mathrm{B}}\right)$, but not in region (1) $\left(H<5 \mathrm{kOe}, T<T_{\mathrm{B}}\right)$. Using the Curie constant $C_{\mathrm{p}}$, we calculated values of $\chi_{\mathrm{p}}^{\mathrm{c}}$ for $T<T_{\mathrm{B}}$ and calculated $\chi_{\mathrm{A}}^{\mathrm{c}}$ values for $T<T_{\mathrm{B}}$ from $\chi_{\mathrm{A}}^{\mathrm{c}}=$ $\chi_{0 \mathrm{~T}}-\chi_{\mathrm{P}}^{\mathrm{c}}$ which should not hold for $T<T_{\mathrm{B}}$ (region (1)) for PIC and Ferritin I. The $\chi_{\mathrm{A}}^{\mathrm{c}}$ values obtained this way represent a marked increase from the $\chi_{\mathrm{A}}$ obtained from the high field magnetization. To illustrate this, several values of the calculated $\chi_{\mathrm{A}}^{\mathrm{c}}$ and $\left(\chi_{\mathrm{A}}^{\mathrm{c}}\right)^{-1}$ are plotted (for $T<T_{\mathrm{B}}$ ) for PIC in Fig. 7(a). It is apparent that in this region there is a third contribution to the initial susceptibility that seems to approach $2 \times$ the magnitude of the antiferromagnetic susceptibility at low temperature. This contribution, we believe, is superantiferromagnetism in which the action of the applied field on the magnetic moment of the surface layers of the particle, which comprise
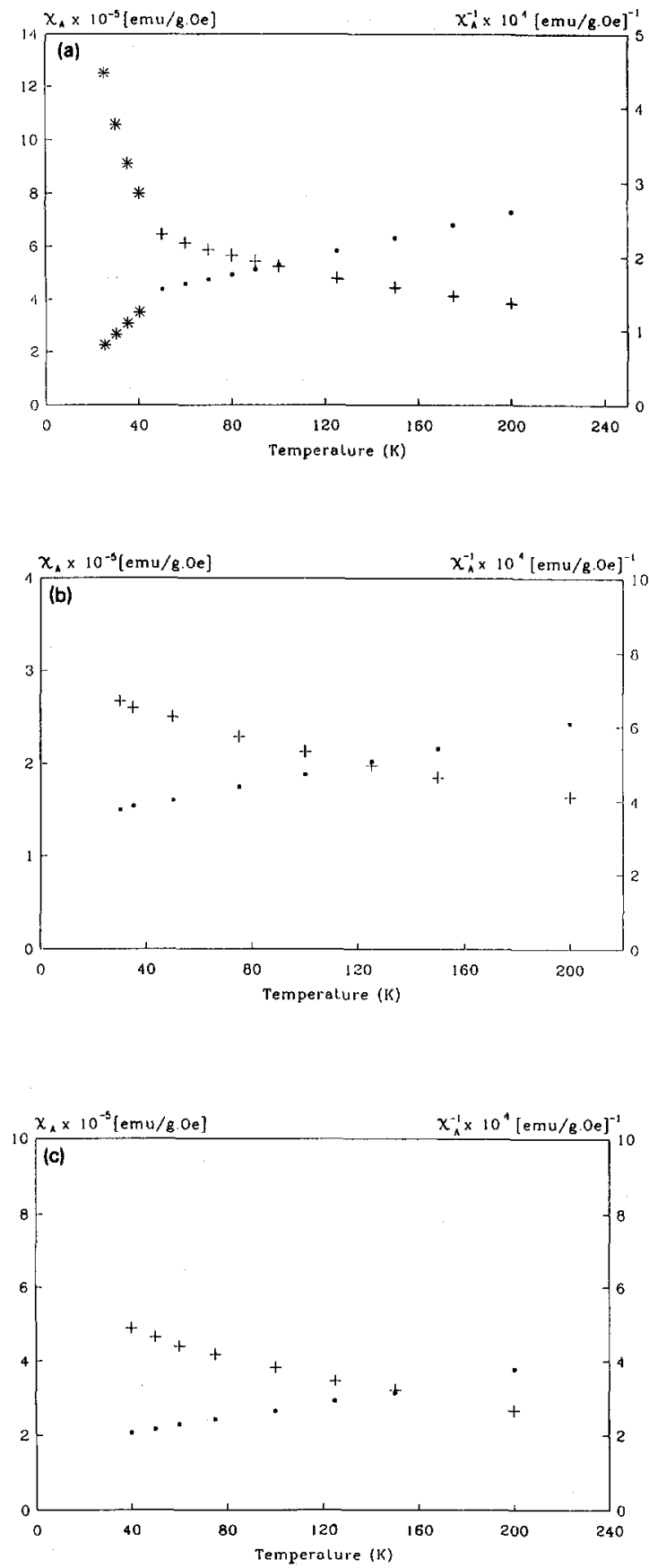

Fig. 7. Antiferromagnetic $\chi_{\mathrm{A}}(T)$ and inverse antiferromagnetic $\chi_{\mathrm{A}}{ }^{-1}(T)$ susceptibilities for: (a) PIC; (b) Ferritin I; (c) Ferritin II. The Curie-Weiss temperature $\Theta$ for: (a) PIC, $\Theta=150 \mathrm{~K}$; (b) Ferritin I, $\Theta=239 \mathrm{~K}$; (c) Ferritin II, $\Theta=160$ K. In (a), * represents the calculated inverse antiferromagnetic susceptibility $\left(\chi_{\mathrm{A}}{ }^{\mathrm{c}}\right)^{-1}$ where $\chi_{\mathrm{A}}{ }^{\mathrm{c}}=\chi_{\mathrm{OT}}-\chi_{\mathrm{P}}{ }^{\mathrm{c}}$ (see text). 
ferromagnetic layer planes of the antiferromagnetic structure, produces a magnetostatic couple, the 'extremity moments' according to Néel [39], which cause a rotation of the direction of antiferromagnetism toward the field direction. The magnitude of this rotation diminishes as one goes from the surface toward the interior of the particle. This would also contribute to or enhance the magnetostriction of such particles and may produce a small deviation of the magnetic anisotropy from being uniaxial. Indeed, the inverse initial susceptibility of PIC and Ferritin I (Figs. 6a, b), indicates an antiferromagnetic interaction between the particles for low temperatures less than $50 \mathrm{~K}$, which we believe is due to the superantiferromagnetic enhancement of the magnetization. This, however, is not as pronounced for Ferritin II (Fig. 6c).

\section{Discussion}

The ferritin cores have been pictured as homogeneous three-dimensional solids with well defined two-dimensional surfaces [45]. However, it has been shown $[13,23]$ that the method by which ferritin carries out its function, which is iron storage, is the intake of $\mathrm{Fe}^{2+}$ ions through the channels in the protein shell. These ions are rapidly oxidized into $\mathrm{Fe}^{3+}$ and are followed by hydrolysis. This process might produce structures with varying degrees of imperfection, i.e. open fractal structures such as those produced by diffusion-limited aggregation (DLA) [23,46]. DLA structures are characterized by highly invaginated surfaces and fluctuations in local density. The ferritin core might thus be a heterogeneous solid with a dense nucleus at the point of attachment to the interior of the protein, and domains of variable size or density that spread out into the cavity like the root structure of a tree growing on a pile of rocks. In other words, the core's structural imperfections may be closer to its surface than its core. According to such DLA core structures, the distribution of blocking temperatures could be ascribed to the domains of differing size or density within a single ferritin molecule. Preferential reduction of the last formed smaller do- mains, in the partially reduced ferritin [II $\left(\mathrm{Fe}^{2+}+\right.$ $\left.\mathrm{Fe}^{3+}\right)$, within a given molecule would shift the average blocking temperature to higher values than for the fully oxidized ferritin $\left[1\left(\mathrm{Fe}^{3+}\right)\right]$ as we have seen in the determination of the regions of superparamagnetism (Fig. 2). The higher average blocking temperatures would correspond to the denser or more crystalline nuclei of the core structure. This may also apply for PIC with its higher density, but PIC also has a larger volume (average diameter $=70 \AA$ ) $[10,17]$ than that for our horse spleen ferritin (average diameter $=62$ Å) [19]. In the superparamagnetic model of Néel [39], the distribution of blocking temperatures reflects the distribution of particle (core) volumes. In our samples this still applies within the same assembly (same density) of particles. The blocking temperatures obtained with Mössbauer studies for Ferritin II [23] are higher than those for Ferritin I, which agree with our results. Similarly, greater blocking temperatures than for Ferritin I were observed for Hemosiderin cores [47], which have smaller average core diameters than regular Ferritin I and higher average core densities. Hemosiderin is a ferritin-like molecule with a large part of the protein shell missing. The higher density could be due to the collapse of the DLA structure.

Mössbauer experiments [23] have suggested that most of the $\mathrm{Fe}^{2+}$ ions in Ferritin I are bound on the surface of the core. Néel [39] has shown that for magnetic single-domain antiferromagnetic particles in this (ferritin and PIC) volume range, the uncompensated spins that produce the magnetic moment of the particle are on the particle's surface. Such a surface that produces the major contribution to the magnetic anisotropy energy of the particle, would greatly affect the particle's magnetic behavior. Given that both ferritin samples have the same volume, and using the determined value for the magnetic moment per particle to estimate the anisotropy constant for both, from the $H_{\mathrm{c}}(T=0)$ values, we get: $K=$ $2.7 \times 10^{5} \mathrm{erg} / \mathrm{cm}^{3}$ for Ferritin $\mathrm{I}$ and $K=3.14 \times$ $10^{5} \mathrm{erg} / \mathrm{cm}^{3}$ for Ferritin II. Hence, the effect of the increase in $K$ by the surface bound $\mathrm{Fe}^{2+}$ ion leads, according to Néel's model (Eq. (6)), to higher blocking temperatures. We note that the 
lower blocking temperature for Ferritin II than that for PIC, even though $K$ (Ferritin II) $\geq$ $K$ (PIC), is due to that $K V$ for Ferritin II is still smaller than the comparable value for PIC due to the latter's larger volume. From either the pair or single-ion model of magnetic anisotropy [48-54] it is apparent how $\mathrm{Fe}^{2+}$ ions bonding to the surface of the Ferritin II core would increase the magnetic anisotropy of the particle, however, such $\mathrm{Fe}^{2+}$ ions may prevent the occurrence of the spin-canting or the 'extremity moments' of Néel, i.e. the magnetostatic couple produced by such antiferromagnetic particles' surfaces in a magnetic field, which gives rise to the superantiferromagnetic effect. However, regardless of the greater values of anisotropy, blocking temperature, density, and maximum superparamagnetic saturation magnetization for PIC than for Ferritin I, the close analogous magnetic behavior of both molecules is evident.

\section{Conclusions}

We have found that ferritin and PIC give a consistent picture of the magnetic behavior of small (within their volume range) antiferromagnetic particles. We believe that PIC has fulfilled the first requirement, mentioned in the introduction, for being a useful 'biomimic' model with which to investigate and compare the LambMössbauer $f$-factor anomaly in ferritin [17]. Moreover, similar anomalous behavior in the Mössbauer spectrum for PIC has recently been confirmed [10].

The implication that ferritin and PIC's magnetic anisotropy may deviate from being strictly uniaxial has been examined [10] using MØrup's collective magnetic excitation theory [55], as manifested by the temperature changes of hyperfine fields (obtained from Mössbauer spectra). Differences [10] between the anisotropy constants obtained from superparamagnetic relaxation spectra, and those obtained from the fit of hyperfine fields, as a function of temperature, using collective magnetic excitation theory, based on uniaxial symmetry, indicated that PIC and ferritin possess magnetic anisotropy energy which is not strictly uniaxial. This, as we have proposed [56-58], is intimately connected with the magnetostriction mechanism, which, we believe, is causing the $f$ factor anomaly.

\section{Acknowledgements}

We wish to thank G.C. Papaefthymiou of the Francis Bitter National Magnet Lab at MIT, Cambridge, Massachusetts, for her cooperation. M.-E.Y. Mohie-Eldin was partially supported by the Office of Naval Research under grant no. N00014-89-J-1779. R.B. Frankel was partially supported by the National Institutes of Health under grant no. 50R1DK36799-05.

\section{References}

[1] K.A. Berg et al., J. Inorg. Biochem. 22 (1984) 125.

[2] L. Michaelis et al., J. Biol. Chem. 148 (1943) 463.

[3] S. Granick and P.F. Hahn, J. Biol. Chem. 155 (1944) 661.

[4] G. Schoffa, Z. Naturf. 20b (1965) 167.

[5] A. Blaise et. al., C.R. Hebd. Seànc. Acad. Sci., Paris, Ser. B 261 (1965) 2310; A. Blaise et al., C.R. Hebd. Seànc. Acad. Sci., Paris, Ser. B 265 (1967) 1077.

[6] A. Blaise and J.L. Girardet, Int. Conf. on Magnetism, Moscow (1973).

[7] J.L. Girardet et al., J. Appl. Phys. 41 (1970) 1002.

[8] T.G. St. Pierre et al., Hyperfine Interactions 29 (1986) 1427.

[9] T.G. St. Pierre et al., J. Magn. Magn. Mater. 69, 276 (1987)

[10] M-E.Y. Mohie-Eldin, R.B. Frankel, L. Gunther and G.C. Papaefthymiou, Hyperfine Interactions, submitted.

[11] J. Yariv et al., Biochem. J. 197 (1981) 171.

[12] E.I. Stiefel and G.D. Watt, Nature 279 (1979) 81.

[13] E.C. Theil, in Advances in Inorganic Biochemistry, vol. 5, eds. E.C. Theil, G.L. Eichorn, and L.G. Marzilli (Elsevier, New York, 1983).

[14] K.M. Towe and W.F. Bradley, J. Colloid Interface Sci. 24 (1967) 384

[15] C.C. Ford et al., Phil. Trans. R. Soc. London B304 (1984) 551.

[16] J.F. Sanders, Mich. Med. 7 (1968) 726; R.W. Nexton et al., Clin. Trials J. 17 (1980) 106; O.W. Crawford, Ill. Med. J. (1970) 157.

[17] M-E.Y. Mohie-Eldin, PhD Thesis, Tufts University, Medford, MA 02155, USA (1992).

[18] U.S. Patent No. 3821192. Process of preparing an IronSaccharide Complex.

[19] J.M. Williams et al., Phys. Med. Biol. 23 (1978) 835. 
[20] T.G. Spiro and P. Saltman, Struct. Bonding (Berlin) 6 (1969) 116; P.J. Murphy et al., J. Colloid Interface Sci. 56 (1976) 312.

[21] A. Müller, Arzneim. Forsch. 17 (1967) 921.

[22] C.W. Childs and J.H. Johnston, Austral. J. Soil Res. 18 (1980) 245.

[23] R.B. Frankel et al., Hyperfine Interactions 33 (1987) 233.

[24] S. Mann et al., J. Molec. Biol. 187 (1986) 47.

[25] L. Néel, Compt. Rend. 228 (1949) 664.

[26] L. Néel, Ann. Geophys. 5 (1949) 99.

[27] L. Néel, Rev. Mod. Phys. 25 (1959) 293.

[28] W.F. Brown, Jr., J. Appl. Phys. 30 suppl. (1959) 1805.

[29] W.F. Brown, Jr., J. Appl. Phys. 34 (1963) 1319.

[30] W.F. Brown, Jr., in Fluctuation Phenomena in Solids, ch. 2 (Academic Press, New York, 1965).

[31] C.P. Bean, J. Appl. Phys. 26 (1955) 1381.

[32] W.J. Schuele, J. Appl. Phys. 36 (1965) 1010.

[33] C.P. Bean and J.D. Livingston, J. Appl. Phys. 30 suppl. (1959) $120 \mathrm{~s}$.

[34] E.P. Wohlfarth, J. Electron. Control 10 (1961) 33

[35] E.P. Wohlfarth, in Magnetism, ch. 7, vol. III, eds. G.T. Rado and H. Suhl (Academic Press, New York, 1963).

[36] E. Kneller and F.E. Lubrosky, J. Appl. Phys. 34 (1963) 656.

[37] K.S. Kaufman et al., Biochim. Biophys. Acta 629 (1980) 522.

[38] T. Shinjo, J. Phys. Soc. Jpn. 21 (1966) 917.

[39] L. Néel, in Low-Temperature Physics, Summer School of Theoretical Physics, Les Hauches (1961), eds. C. Dewitt, B. Dreyfus, and P.G. DeGennes, (Gordon and Breach, New York, 1962).

[40] L. Néel, J. Phys. Soc. Jpn. 17, suppl. B-1 (1962) 676.

[41] C.P. Bean and J.S. Jacobs, J. Appl. Phys. 27 (1956) 1448.
[42] E. Abeledo and P.W. Selwood, J. Appl. Phys. 32 suppl. (1961) $229 \mathrm{~S}$.

[43] D.D. Awschalom, et al., Phys. Rev. Lett., 68 (1992) 3092.

[44] J.S. Jacobs and C.P. Bean, in Magnetism, vol. III, eds. G.T. Rado and H. Suhl (Academic Press, New York, 1963).

[45] P.M. Harrison, in Advances in Inorganic Biochemistry, vol. 5, eds. E.C. Theil, G.C. Eichhorn, and L.G. Marzilli, (Elsevier, New York, 1983).

[46] H.E. Stanley and N. Ostrowsky, eds., On Growth and Form (Kluwer, Hingham, MA, 1985).

[47] S.H. Bell et al., Biochem. Biophys. Acta 787 (1984) 227.

[48] J.H. Van Vleck, Phys. Rev. 50 (1937) 376; J. Phys. Radium 12 (1951) 262.

[49] T. Moriya, Phys. Rev. Lett. 4 (1960) 228; Phys. Rev. 120 (1960) 91.

[50] T. Nagamiya et al., Adv. Phys. Part I, 6 (1955) 4.

[51] F. Bloch and G. Gentile, Z. Phys. 70 (1931) 395.

[52] K. Yosida and M. Tachiki, Prog. Theor. Phys. (Kyoto) 17 (1957) 331.

[53] W.P. Wolf, Phys. Rev. 108 (1957) 1152.

[54] J.C. Slonczewski, Phys. Rev. 110 (1958) 1341.

[55] S. Mørup and H. Topsøe, Appl. Phys. 11 (1976) 63; S. Morup et al., J. Physique 37 (1976) C6-287.

[56] M.-E.Y. Mohie-Eldin and L. Gunther, in Proc. Int. Workshop on Studies of Magnetic Properties of Fine Particles and Their Relevance to Materials Science, Rome, Italy, eds. J.L. Dormann and D. Fiorani (Elsevier, Amsterdam, 1992), p. 403.

[57] M.-E.Y. Mohie-Eldin and L. Gunther, J. Magn. Magn. Mater. 127 (1993) 346.

[58] L. Gunther and M.-E.Y. Mohie-Eldin, J. Magn. Magn. Mater. 129 (1994) 334. 